\title{
Clinical Outcomes of Extracorporeal Shock Wave Therapy in Patients With Secondary Lymphedema: A Pilot Study
}

\author{
Hasuk Bae, MD, Ho Jeong Kim, MD \\ Department of Rehabilitation Medicine, Ewha Womans University School of Medicine, Seoul, Korea
}

Objective To investigate the clinical effect of extracorporeal shock wave therapy (ESWT) in patients with secondary lymphedema after breast cancer treatment.

Methods In a prospective clinical trial, ESWT was performed consecutively 4 times over two weeks in 7 patients who were diagnosed with stage 3 secondary lymphedema after breast cancer treatment. Each patient was treated with four sessions of ESWT (0.056-0.068 $\mathrm{mJ} / \mathrm{mm}^{2}, 2,000$ impulses). The parameters were the circumference of the arm, thickness of the skin and volume of the arm. We measured these parameters with baseline values before ESWT and repeated the evaluation after each ESWT treatment. Subjective data on skin thickness, edema and sensory impairment were obtained using a visual analogue scale (VAS).

Results The mean volume of the affected arm after four consecutive ESWT was significantly reduced from 2,332 to $2,144 \mathrm{~mL}(\mathrm{p}<0.05)$. The circumference and thickness of the skin fold of the affected arm were significantly decreased after the fourth ESWT $(\mathrm{p}<0.05)$. The three VAS scores were significantly improved after the fourth ESWT. Almost all patients were satisfied with this treatment and felt softer texture in their affected arm after treatment.

Conclusion ESWT is an effective modality in the treatment of stage 3 lymphedema after breast cancer treatment. ESWT reduced the circumference and the thickness of arms with lymphedema and satisfied almost all patients with lymphedema. Therefore, this treatment provides clinically favorable outcome to patients with breast cancerrelated lymphedema.

Keywords Lymphedema, Breast neoplasms, High-energy shock waves, Physiotherapy

Received October 19, 2011; Accepted September 24, 2012

Corresponding author: Ho Jeong Kim

Department of Rehabilitation Medicine, Ewha Womans University School of Medicine, 1071 Anyangcheon-ro, Yangcheon-gu, Seoul 158710, Korea

Tel: +82-2-2650-6020, Fax: +82-2-2650-5145, E-mail: hoyabbun5050@ hanmail.net

(c) This is an open-access article distributed under the terms of the Creative Commons Attribution Non-Commercial License (http://creativecommons. org/licenses/by-nc/3.0) which permits unrestricted noncommercial use, distribution, and reproduction in any medium, provided the original work is properly cited.

Copyright $\odot 2013$ by Korean Academy of Rehabilitation Medicine

\section{INTRODUCTION}

Lymphedema is a chronic and progressive condition resulting from an abnormality or damage to the lymphatic system. It is marked by an abnormal increase of tissue proteins, edema, chronic inflammation, and fibrosis. Secondary lymphedema is caused by multiple factors related with lymphatic stasis, such as tumor lymph node infiltration, lymph node dissection, radiotherapy, trauma, and infection [1]. Upper limb lymphedema occurs in $24 \%-$ $49 \%$ of the cases with total mastectomy and in $2.4 \%-49 \%$ 
of the cases with axillary lymph node dissection [2,3]. In Korea, upper limb secondary lymphedema has been reported in $22 \%$ of patients after breast cancer therapy [4]. Lymphedema occurs when there is an imbalance due to reduced lymph transport capacity which leads to interstitial fluid and protein accumulation [1]. It further leads to chronic inflammation and fibrosis caused by the secondary proliferation of neutrophils, macrophages, and fibroblasts and accumulation of collagen. One of the most common forms of treatment is complex decongestive physical therapy (CDPT) $[5,6]$. CDPT involves various techniques such as scrupulous skin care, manual lymphatic drainage, external compression device, exercise to improve lymphatic movement by well-trained therapists, and intensive patient education. Studies have shown a decrease of around $21 \%-60 \%$ of lymphedema volume with CDPT $[4,7,8]$. The efficacy of CDPT varies depending on the characteristics of the patient, and worse compliance is associated with a reincrease in lymphedema volume [5,9-11]. However, CDPT is less effective in stage 3 lymphedema, characterized by fibrotic and unresponsive tissue, than stage 1 and 2 lymphedema $[9,12]$. Extracorporeal shock wave therapy (ESWT) has been in widespread use in orthopedics. ESWT has a positive influence on calcifying tendinitis of the shoulder, epicondylitis of the elbow, and plantar fasciitis. Recent studies have demonstrated that ESWT helps angiogenesis and decreases inflammation [13-15] in the human body and helps lymphangiogenesis in animals [16].

Previous studies which proved reduction of inflammation and lymphagiogenesis did not include human applicants. In the present study, we aimed to demonstrate the effect of ESWT on stage 3 lymphedema by subjective and objective measure.

\section{MATERIALS AND METHODS}

\section{Subjects}

Subjects were enrolled after giving written informed consent for the study which was approved by the institutional review board. Recruitment was undertaken from the population of outpatients, who had upper extremity lymphedema, from the rehabilitation department. To determine the presence of lymphedema objectively, a limb-to-limb difference of $2 \mathrm{~cm}$ or more at a single measurement site and lymphatic obstruction confirmed by lymphoscintigraphy were used [12]. Only seven patients who were diagnosed with stage 3 lymphedema advanced dermatofibrotic change and had been resistant to CDPT were included. Evidence of metastasis on the affected arm, neuropathy, anomaly of vessel, infection, hyperventilation, polyneuropathy, pregnancy constituted the exclusion criteria.

\section{Methods}

ESWT was carried out twice a week for two weeks using electromagnetic type Dornier AR2 (Dornier MedTech $\mathrm{GmBH}$, Wessling, Germany), and the stimulus was given on the treatment site according to the patient's tolerance, 2,000 times in one session with an energy of 0.056-0.068 $\mathrm{mJ} / \mathrm{mm}^{2}$. Stimulus was applied 1,000 times to the most fibrotic lesion felt by the examiner's palpation, and the other 1,000 times was applied to other less fibrotic lesion. CDPT or pneumatic compression was not successful on these subjects in the past. Therefore, we divided the subjects into two groups by their opinion; four patients were treated by manual lymphatic massage and pneumatic compression with ESWT, and the rest three patients were treated with ESWT only.

All patients were evaluated by subjective and objective measurements before treatment and after four session of treatment. The subjective measurements are hardness of the skin, edema of the involved upper extremity, and sensory impairment. Each of them was measured by a visual analogue scale (VAS), with ten points being the most severe degree. Objective measurements were the volume of the upper extremity, thickness of the skin, and circumference of the upper extremity. Measurement of volume was a direct technique and consisted of a derivation of Archimedes's principle. Before measurement of volume, the patients wiped their upper extremity. The entire involved arm, from the hand to the axilla, was immersed in fluid and compared to the uninvolved site. This was done between the hours of 1 and 5 PM before management and at the same time after treatment. The circumference of the upper extremity was measured $10 \mathrm{~cm}$ below the axilla, $10 \mathrm{~cm}$ above the elbow, $7 \mathrm{~cm}$ below the elbow, and 7 $\mathrm{cm}$ above the wrist [17]. The contralateral upper extremity was measured at the same levels and the values were compared with the involved side. Skin fold thickness was measured at the same four levels as those for circumference measurement. A skin fold caliper was used and the 
measured values were compared with the uninvolved side.

\section{Statistical analysis}

Comparisons of measurements between before and after treatment were performed using Wilcoxon's singed rank test. Analyses were performed using SPSS ver. 15.0 (SPSS Inc., Chicago, IL, USA) and p-values under 0.005 were considered significant.

\section{RESULTS}

Seven patients who were diagnosed with upper limb lymphedema after breast cancer operation were referred for four sessions of ESWT. Any side effect was reported during the four sessions. Subjects were women who were managed by modified radical mastectomy with adjuvant chemotherapy and/or radiation therapy. The mean age was $52 \pm 9.9$ years and the mean length of time suffering from lymphedema was $25.3 \pm 12.7$ months. All patients were stage 3 lymphedema and had been treated by physical therapy. Three patients who had been treated in our hospital for three months did not show any decrease in skin hardness or edema as well as objective reduction of arm circumference. The other four patients had been treated at other hospitals and did not experience subjective improvement. In this study, four patients were treated by ESWT, manual lymphatic drainage by a physician, and pneumatic compression. The other three patients refused physical therapy except ESWT (Table 1).

Reduction of lymphedema volume was shown after four c sessions of ESWT in all patients. The mean volume of the affected upper extremity before treatment was

Table 1. General characteristics of the subjects $(n=7)$

\begin{tabular}{lc}
\hline \multicolumn{1}{c}{ Characteristic } & Value \\
\hline Age $(\mathrm{yr})$, mean \pm SD & $52 \pm 9.9$ \\
Female & 7 \\
Duration of lymphedema $(\mathrm{mo})$, mean \pm SD & $25.3 \pm 12.7$ \\
Lymphedema stage 3 & 7 \\
Chemotherapy & 7 \\
Radiotherapy & 7 \\
Combination physical therapy $^{\mathrm{a})}$ & 4 \\
\hline
\end{tabular}

SD, standard deviation.

${ }^{\text {a) }}$ Physical therapy, manual massage and pneumatic compression by a physical therapist.
$2,332.9 \pm 580.9 \mathrm{~mL}$ and after treatment was $2,144.3 \pm 544.4$ $\mathrm{mL}$. The reduction volume was $188.6 \mathrm{~mL}$, which was determined to be significant $(\mathrm{p}=0.018)$. The mean reduction rate was $37.23 \%$ (Fig. 1). A comparison of two groups, which were treated simultaneously with physical therapy or without, revealed the reduction rate of $39.46 \%$ and $34.25 \%$, respectively. The difference was $5 \%$, but it was not significant. The means of the circumference of the lymphedema involved upper limb after treatment were significantly decreased from 31.94 to $30.97 \mathrm{~cm}$ at $10 \mathrm{~cm}$ below the axilla ( $\mathrm{p}=0.042$ ), from 30.12 to $29.57 \mathrm{~cm}$ at 10 $\mathrm{cm}$ above the elbow ( $\mathrm{p}=0.018$ ), from 26.43 to $25.62 \mathrm{~cm}$ at $7 \mathrm{~cm}$ below the elbow ( $\mathrm{p}=0.027$ ), and from 20.74 to 19.82 $\mathrm{cm}$ at $7 \mathrm{~cm}$ above the wrist ( $\mathrm{p}=0.018)$. The site of the biggest decrease in circumference after treatment was different in each patient; five of seven patients were below the elbow and the other two were above the elbow. The mean decrease in circumference was $1.1 \mathrm{~cm}$, ranging from 0.4 to $1.7 \mathrm{~cm}$. The means of skin thickness after treatment were significantly decreased from 46.34 to $44.57 \mathrm{~mm}$ at $10 \mathrm{~cm}$ below the axilla ( $\mathrm{p}=0.016$ ), from 45.14 to $41.71 \mathrm{~mm}$ at $10 \mathrm{~cm}$ above the elbow ( $\mathrm{p}=0.016)$, from 43.43 to 35.71 $\mathrm{mm}$ at $7 \mathrm{~cm}$ below the elbow ( $\mathrm{p}=0.018)$, and from 30.29 to $24.14 \mathrm{~mm}$ at $7 \mathrm{~cm}$ above the wrist $(\mathrm{p}=0.026)$. The site of the biggest decrease in skin fold thickness after treatment was also different. The mean decrease in skin fold was 7.7 $\mathrm{mm}$, ranging from 4 to $16 \mathrm{~mm}$; six of seven patients were below the elbow and the other one was above the elbow (Fig. 2). The mean skin hardness measured by VAS was 7.6 before treatment and 5 after the four session of ESWT. This decrease was determined to be significant $(\mathrm{p}=0.028)$.

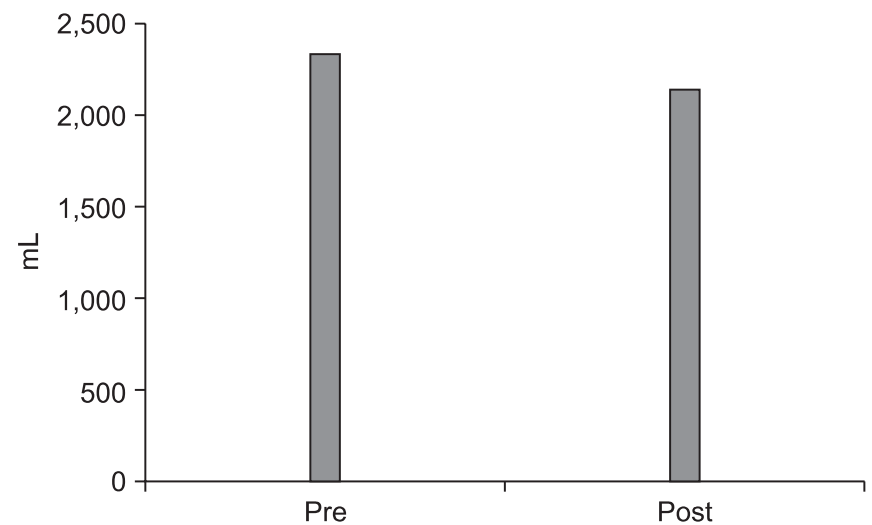

Fig. 1. The volume of the affected upper extremity after four consecutive extracorporeal shock wave therapy (ESWT) was reduced significantly $(\mathrm{p}<0.05)$. After ESWT, the mean of volume drop was $37.23 \%$. 

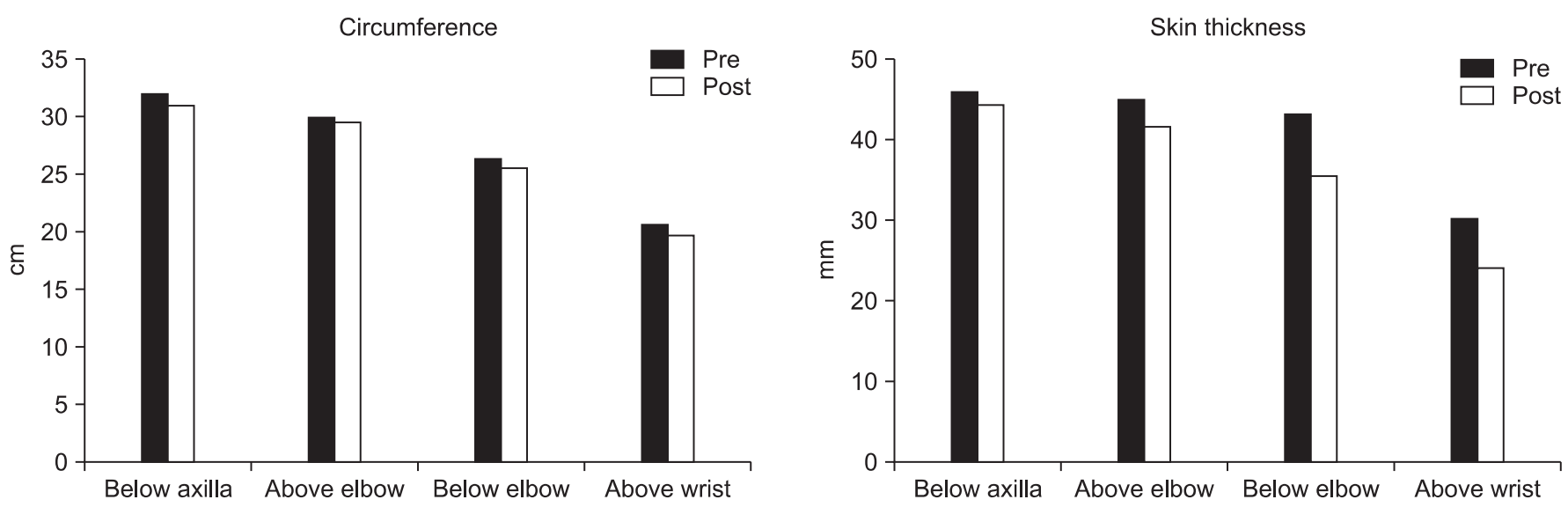

Fig. 2. Circumference and thickness of the skin fold of the affected upper extremity were significantly decreased after four extracorporeal shock wave therapy $(\mathrm{p}<0.05)$. All the circumferences and skin folds below the axilla, above elbow, below elbow, and above the wrist were significantly reduced.

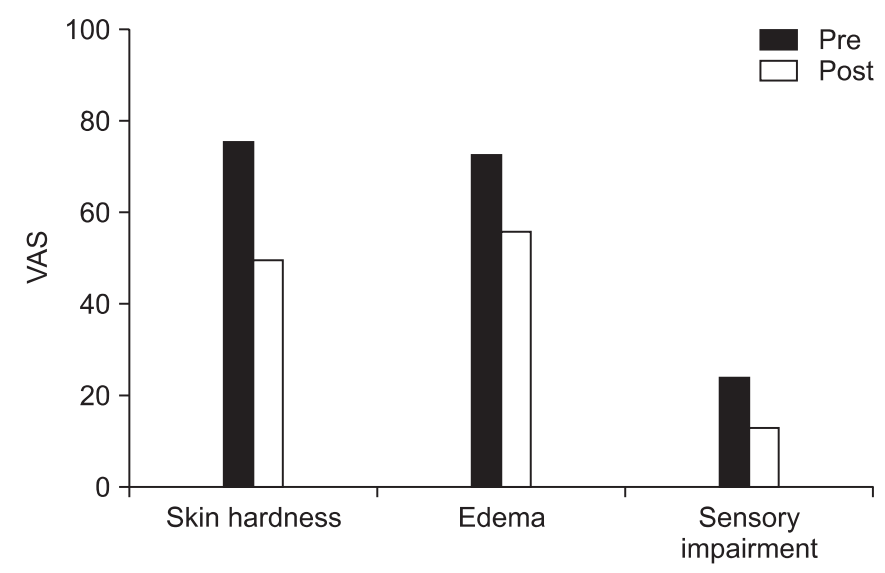

Fig. 3. Visual analogue scales (VASs) of skin hardness, edema, and sensory loss were significantly improved after the fourth extracorporeal shock wave therapy $(\mathrm{p}<0.05)$.

The mean subjective edema was significantly decreased from 7.3 to $5.6(\mathrm{p}=0.027)$. Sensory impairment was also significantly decreased from 2.4 to 1.3 ( $\mathrm{p}=0.046$ ) (Fig. 3). Side effects such as hematoma, subcutaneous hemorrhage and others did not occur.

\section{DISCUSSION}

Lymphedema is the accumulation of protein rich interstitial fluid as a result of impaired lymphatic function. Chronic lymph stasis typically stimulates an increase in the number of fibroblasts, adipocytes, and keratinocytes in the skin, in addition to promoting a large infiltration of neutrophils. This cellular and molecular milieu is a profound stimulus to collagen deposition in the integument
[12]. Stage 3 lymphedema, which is characterized fibrotic and unresponsive tissue, becomes less effective to manual lymphatic drainage and pneumatic compression. Williams et al. [6] showed $10 \%$ reduction of lymphedema volume by manual lymphatic drainage. Szuba et al. [18] reported $45 \%$ reduction in lymphedema volume. However, these effects were shown in just stage 1-2 lymphedema. With progression into the latter grades, treatment becomes more difficult and is likely to produce a lessthan-optimal outcome $[6,9,18-22]$.

This study was a pilot study which evaluated the effectiveness of ESWT as a treatment technique for the management of stage 3 secondary lymphedema. Clinical measurements such as the volume and circumference of the upper limb and skin fold thickness were changed between before and after treatment. All subjects who were treated with four sessions of ESWT showed a significant reduction in lymphedema volume, limb circumference, and skin fold thickness. In this study, all participants were stage 3 lymphedema patients and showed significant volume reduction $(37.23 \%)$. Volume reduction in stage 3 lymphedema is superior or equal to volume reduction $(10 \%-45 \%)$ in stage $1-2$ lymphedema patients receiving CDPT.

Christ et al. [23] confirmed the improvement of skin elasticity in the treatment of cellulitis and connective tissue weakness by means of ESWT. Patients in their study received treatment for 6 therapy sessions and were followed up for 3 months. It was observed that the network of collagen/elastic fibers in the dermis and subcutis became denser and measurably firmer. In the parallel 
biomechanical examinations, reduced oxidative stress by ESWT was shown by this study by means of increased lipolysis and by the release of toxic aldehydic products of lipid. Christ et al. [23] improved collagen synthesis and showed measurable and visible improvement of skin condition. This study also confirmed the decrease of orange pill sign which was shown in stage 3 lymphedema and other measurements of skin conditions including skin fold thickness and skin hardness. This may indicate improvement of skin elasticity and connective tissue strength. ESWT was applied to patients affected by systemic sclerosis by Tinazzi et al. [15]. ESWT resulted in an improvement of VAS and Rodnan skin score for skin wellness and in increased endothelial progenitor cells and circulatory endothelial cells [15]. Schaden et al. [13] and Mariotto et al. [14] demonstrated that ESWT promote angiogenesis, decrease neutrophils and inflammation, and decrease the number of adipocytes. The subjective change of upper limb in this study would be the result of ESWT promoting reduction of inflammatory cells, reconditioning of skin tissue and improvement of lymphatic drainage. Low-energy ESWT induces therapeutic lymphangiogenesis by up-regulating vascular endothelial growth factor $\mathrm{C}$ and basis fibroblast growth factor, and by improving lymphedema in a rat model [16]. Therefore, the improvement of lymphedema in our study could be the result of lymphangiogenesis. The group receiving ESWT plus manual lymphatic drainage and pneumatic compression showed superior volume reduction of $5 \%$ compared with the ESWT alone group, but the difference was not significant ( $p=0.724)$. It is thought that ESWT helps the effect of lymphedema treatment for manual lymphatic drainage and pneumatic compression.

The limitation of the current study is the small number of patients divided three groups treated by ESWT and four patients managed by ESWT with other traditional physical therapy. It includes patients, with objective assessment of improvement in the past. If patients had objective improvement but did not subjectively feel satisfied with the past treatment, the therapeutic effect could be due to the placebo effect as well as ESWT itself. Therefore, a further clinical study including more patients and long-term follow-up will be needed to introduce ESWT into the management strategy for the treatment of lymphedema. Although all patients showed improvement of lymphedema in this study, we did not investigate and classify the onset and other risk factors of lymphedema. In this study, ESWT was applied to the site which was already fibrosed and the site at the stage of the beginning fibrosis. This study shows the effect on the improvement of fibrosis and inhibition of fibrosis progression. However, this study did not evaluate objective and subjective measurements regarding the fibrosis and did not subdivide which factors of ESWT affect the sclerosis of lymphedema. We need further consideration before suggesting the clinical use of ESWT on lymphedema. Also, we could not find the mechanism and the obvious effect of ESWT by additional or independent treatment of lymphedema. Therefore, a further clinical and animal study will be needed to identify the effect and mechanism of ESWT by means of lymphedema treatment.

ESWT is an effective modality in the treatment of stage 3 lymphedema after breast cancer operation. ESWT with or without other traditional treatment modalities significantly reduced the objective and subjective measurements of lymphedema. Therefore, this noninvasive treatment provides clinically favorable outcome to patients with breast cancer-related lymphedema.

\section{CONFLICT OF INTEREST}

No potential conflict of interest relevant to this article was reported.

\section{REFERENCES}

1. Warren AG, Brorson H, Borud LJ, Slavin SA. Lymphedema: a comprehensive review. Ann Plast Surg 2007;59:464-72.

2. Hinrichs CS, Watroba NL, Rezaishiraz H, Giese W, Hurd T, Fassl KA, et al. Lymphedema secondary to postmastectomy radiation: incidence and risk factors. Ann Surg Oncol 2004;11:573-80.

3. Querci della Rovere G, Ahmad I, Singh P, Ashley S, Daniels IR, Mortimer P. An audit of the incidence of arm lymphoedema after prophylactic level I/II axillary dissection without division of the pectoralis minor muscle. Ann R Coll Surg Engl 2003;85:158-61.

4. Kim KS, Ko HY. Upper extremity lymphedema secondary to breast cancer. J Korean Acad Rehabil Med 1992;16:94-100.

5. Casley-Smith JR, Casley-Smith JR. Treatment of 
lymphedema by complex physical therapy, with and without oral and topical benzopyrones: what should therapists and patients expect. Lymphology 1996;29:76-82.

6. Williams AF, Vadgama A, Franks PJ, Mortimer PS. A randomized controlled crossover study of manual lymphatic drainage therapy in women with breast cancer-related lymphoedema. Eur J Cancer Care (Engl) 2002;11:254-61.

7. Ko DS, Lerner R, Klose G, Cosimi AB. Effective treatment of lymphedema of the extremities. Arch Surg 1998;133:452-8.

8. Devoogdt N, Van Kampen M, Geraerts I, Coremans T, Christiaens MR. Different physical treatment modalities for lymphoedema developing after axillary lymph node dissection for breast cancer: a review. Eur J Obstet Gynecol Reprod Biol 2010;149:3-9.

9. Yamamoto R, Yamamoto T. Effectiveness of the treatment-phase of two-phase complex decongestive physiotherapy for the treatment of extremity lymphedema. Int J Clin Oncol 2007;12:463-8.

10. Boris M, Weindorf S, Lasinkski S. Persistence of lymphedema reduction after noninvasive complex lymphedema therapy. Oncology(Williston Park) 1997;11:99-109.

11. Casley-Smith JR, Morgan RG, Piller NB. Treatment of lymphedema of the arms and legs with 5,6-benzo[alpha]-pyrone. N Engl J Med 1993;329:1158-63.

12. Korpan MI, Crevenna R, Fialka-Moser V. Lymphedema: a therapeutic approach in the treatment and rehabilitation of cancer patients. Am J Phys Med Rehabil 2011;90(5 Suppl 1):S69-75.

13. Schaden W, Thiele R, Kolpl C, Pusch M, Nissan A, Attinger CE, et al. Shock wave therapy for acute and chronic soft tissue wounds: a feasibility study. J Surg Res 2007;143:1-12.

14. Mariotto S, Cavalieri E, Amelio E, Ciampa AR, de Prati AC, Marlinghaus E, et al. Extracorporeal shock waves: from lithotripsy to anti-inflammatory action by no production. Nitric Oxide 2005;12:89-96.

15. Tinazzi E, Amelio E, Marangoni E, Guerra C, Puccetti A, Codella OM, et al. Effects of shock wave therapy in the skin of patients with progressive systemic sclerosis: a pilot study. Rheumatol Int 2011;31:651-6.

16. Serizawa F, Ito K, Matsubara M, Sato A, Shimokawa $\mathrm{H}$, Satomi S. Extracorporeal shock wave therapy induces therapeutic lymphangiogenesis in a rat model of secondary lymphoedema. Eur J Vasc Endovasc Surg 2011;42:254-60.

17. Didem K, Ufuk YS, Serdar S, Zumre A. The comparison of two different physiotherapy methods in treatment of lymphedema after breast surgery. Breast Cancer Res Treat 2005;93:49-54.

18. Szuba A, Achalu R, Rockson SG. Decongestive lymphatic therapy for patients with breast carcinomaassociated lymphedema. A randomized, prospective study of a role for adjunctive intermittent pneumatic compression. Cancer 2002;95:2260-7.

19. Lee BB, Kim YW, Kim DI, Hwang JH, Laredo J, Neville R. Supplemental surgical treatment to end stage (stage IV-V) of chronic lymphedema. Int Angiol 2008;27:38995.

20. Andersen L, Hojris I, Erlandsen M, Andersen J. Treatment of breast-cancer-related lymphedema with or without manual lymphatic drainage: a randomized study. Acta Oncol 2000;39:399-405.

21. Casley-Smith JR, Boris M, Weindorf S, Lasinski B. Treatment for lymphedema of the arm: the CasleySmith method. A noninvasive method produces continued reduction. Cancer 1998;83(12 Suppl):2843-60.

22. Karadibak D, Yavuzsen T, Saydam S. Prospective trial of intensive decongestive physiotherapy for upper extremity lymphedema. J Surg Oncol 2008;97:572-7.

23. Christ C, Brenke R, Sattler G, Siems W, Novak P, Daser A. Improvement in skin elasticity in the treatment of cellulite and connective tissue weakness by means of extracorporeal pulse activation therapy. Aesthet Surg J 2008;28:538-44. 